\title{
Bacillus subtilis AISLADA DE CUTÍCULAS DE HORMIGAS QUE ANIDAN EN COLMENAS COMO ANTIFÚNGICO DE HONGOS PATÓGENOS DE ABEJAS
}

\section{Bacillus subtilis ISOLATED FROM CUTICLES OF ANTS NESTING IN HIVES AS ANTIFUNGAL AGAINST FUNGAL PATHOGENS OF BEES}

\author{
Gisela Beatriz Ruiz $^{1 \mathrm{a} *}$ Rosa Milagro Retamoso ${ }^{1 \mathrm{~b}}$ y Marcelo Benítez Ahrendts ${ }^{1 \mathrm{c}}$ \\ ${ }^{1 a}$ Laboratorio de Microbiología y Salud Apícola, Facultad de Ciencias Agropecuarias, Universidad \\ Nacional de Jujuy, Alberdi 47. San Salvador de Jujuy, Jujuy, Argentina \\ https://orcid.org/0000-0002-7952-5357 \\ ${ }^{1 b}$ Laboratorio de Microbiología y Salud Apícola, Facultad de Ciencias Agropecuarias, Universidad \\ Nacional de Jujuy, Alberdi 47. San Salvador de Jujuy, Jujuy, Argentina \\ ${ }^{1 c}$ Laboratorio de Microbiología y Salud Apícola, Facultad de Ciencias Agropecuarias, Universidad \\ Nacional de Jujuy, Alberdi 47. San Salvador de Jujuy, Jujuy, Argentina \\ https://orcid.org/0000-0001-8479-8985 \\ * Autor para correspondencia E-mail: giselaruiz574@gmail.com
}

\section{RESUMEN}

La presencia de hormigas en los apiarios ha sido considerada durante mucho tiempo un problema para la apicultura. En la producción apícola es muy importante el aspecto sanitario de las colmenas, ya que las abejas melíferas son afectadas por virus, bacterias, hongos y parásitos. Penicillium, Aspergillus y Fusarium son micotoxigénicos con una amplia distribución en el mundo. Se ha demostrado la presencia de estos hongos en el tubo digestivo de las abejas y en la miel. Una de las bacterias más estudiadas como antagonistas es Bacillus. El objetivo del estudio fue determinar la actividad antifúngica de Bacillus subtilis frente Aspergillus sección nigri y Fusarium solani. Se realizó el aislamiento en Agar nutritivo de B. subtilis de cutículas de hormigas de colmenares de los valles templados de la provincia de Jujuy, Argentina. Los hongos fueron provistos por el cepario del Laboratorio de Microbiología Agrícola y Sanidad Apícola y Meliponícola de la Facultad de Ciencias Agrarias, UNJu. Las pruebas de antagonismo se realizaron en placas con medio agar papa dextrosa con explantes de los hongos frente a Bacillus subtilis incubadas a $28^{\circ} \mathrm{C}$ durante una semana. Se registraron los diámetros de las colonias y se determinó el porcentaje de inhibición a los 5,10 y 15 días. Los porcentajes de inhibición fueron mayores a los 5 días de incubación. El porcentaje de inhibición fue mayor al $50 \%$ en Aspergillus sección nigri y mayor al $65 \%$ en Fusarium solani. Bacillus subtilis mostró actividad antifúngica contra los hongos patógenos de abejas en estudio.

Palabras clave: Cepa bacteriana, Inhibición, formícidos, apiarios, Aspergillus sección nigri, Fusarium solani.

\section{ABSTRACT}

The presence of ants in apiaries has long been considered a problem for beekeeping. The health of the hives is a key issue in beekeeping since honey bees are affected by viruses, bacteria, fungi and parasites. Penicillium, Aspergillus and Fusarium are mycotoxigenic fungi, which are widely distributed throughout the world. The presence of these fungi has been observed in both the digestive 
tract of bees and honey. One of the most studied bacteria as antagonists is Bacillus. The objective of the study was to determine the antifungal activity of Bacillus subtilis against Aspergillus section nigri and Fusarium solani. The isolation was carried out in nutritive agar of $B$. subtilis from cuticles of ants from apiaries of the temperate valleys of the province of Jujuy, Argentina. The fungal strains were obtained from the strain collection of the Laboratory of Agricultural Microbiology and Beekeeping/ Stingless beekeeping Health of the Faculty of Agrarian Sciences, UNJu. The antagonism tests were performed on plates with potato dextrose agar medium with explants of the fungi against Bacillus subtilis incubated at $28^{\circ} \mathrm{C}$ for one week. Colony diameters were recorded, and the percentage of growth inhibition was determined at 5, 10 and 15 days. The inhibition percentages were higher after 5 days of incubation. The inhibition percentage was greater than $50 \%$ in Aspergillus section nigri and greater than $65 \%$ in Fusarium solani. Bacillus subtilis showed antifungal activity against the pathogenic fungi of bees under study.

Key words: Bacterial strain, Inhibition, formicids, apiaries, Aspergillus section nigri, Fusarium solani.

\section{INTRODUCCIÓN}

La presencia de hormigas en los apiarios indistintamente de la especie a la que pertenecen, ha sido reportada principalmente por competir por el alimento de las abejas, polen, miel y además por robar las crías del interior y preceder a las abejas en las colmenas, que resulta en el debilitamiento de la misma y en impactos negativos en la eficiencia de la polinización y en la producción de miel de las abejas (Calle-Barrientos y Agudelo-Valencia, 2016). A pesar de esto no existe antagonismo o competencia entre las abejas y las hormigas, en ocasiones construyen nidos en las proximidades o dentro de las colmenas con el fin de hallar un lugar abrigado donde establecerse (Ruiz y Benítez, 2018). De acuerdo a Valega (2016) y Ricci (2005) las hormigas suelen aprovechar el calor y la humedad de la colmena para incubar sus huevos, utilizándolas como refugios temporales o permanentes y no resultan perjudiciales en la producción de miel.

Apis mellifera L. (Orden Hymenoptera, Familia Apidae) es afectada por distintos agentes etiológicos como virus, bacterias, hongos y parásitos, que en su mayoría ocasionan daños considerables en la apicultura favoreciendo la aparición de enfermedades (Verde Jiménez et al., 2013). Las hormigas suelen tener microorganismos adheridos a sus cutículas. La presencia de diferentes microorganismos adheridos a las cutículas de hormigas en colmenas de A. mellifera pueden influir directa o indirectamente en la salud de las abejas (Ruiz et al., 2019).

Entre los hongos oportunistas que causan enfermedades en las colmenas, se encuentran las especies del género Aspergillus. Son cosmopolitas, corresponden a uno de los principales grupos de hongos contaminantes del ambiente. Juegan un rol ecológico importante en los ciclos del Carbono y Nitrógeno, por lo que pueden ser aislados de cualquier sustrato que contenga materia orgánica y humedad, como suelo, restos vegetales en descomposición, alimentos, etc. Los propágulos del género Aspergillus pueden permanecer en suspensión por periodos prolongados y contaminar cualquier superficie (López, 2014). Una enfermedad micótica que afecta a las abejas adultas y a la cría es la Aspergilosis, que ocasiona la muerte y momificación. La aspergilosis o "cría de piedra" es la enfermedad producida por varias especies del género Aspergillus con mayor frecuencia $A$. flavus, A. niger y $A$. fumigatus, los que afectan a las crías de cualquier edad y a las abejas adultas (FAO, 2018). Las especies de Fusarium son micotoxinógenas con amplia distribución en el mundo que pueden afectar al hombre y a los animales (Serrano-Colli y Cardona-Castro, 2015). Fusarium tiene varios factores de virulencia incluidas las micotoxinas (fusarinas) que inhiben la inmunidad celular y pueden causar daño tisular (Martínez-Hernández y Callejas-Chavero, 2015). Se ha evidenciado la presencia de los hongos Penicillium, Aspergillus y Fusarium en el tubo digestivo de las abejas, así como en la miel y otras provisiones de estos insectos (Ramírez Del Jesús, 2016).

Por otro lado, Bacillus subtilis es un biocontrolador con amplio espectro antibiótico; los metabolitos que producen son supresores efectivos de algunos patógenos de plantas, como Fusarium, Pythium, Phytophthora, Rhizoctonia, Sclerotinia, Septoria y Verticillium (VillarrealDelgado et al., 2018). Se ha encontrado que las bacterias del género Bacillus tienen un gran potencial como antagonistas, principalmente debido a la gran cantidad de enzimas líticas, antibióticos y otras sustancias con actividad biocida que son capaces de producir efectos de control sobre varias especies de organismos fitopatógenos (Castillo-Reyes et al., 2015; Villarreal-Delgado et al., 2018). El objetivo del presente estudio fue determinar el efecto biocontrolador de Bacillus subtilis aisladas de cutículas de hormigas que habitan las colmenas sobre Aspergillus sección nigri y Fusarium 
solani que resultan patógenas para las abejas. Evidenciando de esta manera la relación existente entre hormigas y abejas.

\section{MATERIALES Y MÉTODOS}

\section{Aislamiento de Bacillus subtilis}

Para el aislamiento de esta bacteria primero se colectaron hormigas que se encontraban dentro de colmenas de un apiario perteneciente a socios de la Cooperativa de Productores Apícolas de Jujuy Ltda. (COPAJ), ubicado en la localidad de

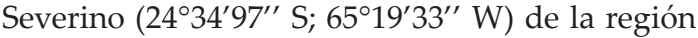
de los Valles Templados de la provincia de Jujuy, Argentina. El material recolectado se colocó en frascos estériles y se llevó al Laboratorio de Microbiología Agrícola y Sanidad Apícola de la Facultad de Ciencias Agrícolas (UNJu). Para el aislamiento se suspendieron cinco ejemplares de hormigas de la misma especie en una solución de 1,5 mL de peptona al 10\% en tubos de Eppendorf, estos fueron agitados en Vórtex por cinco minutos. Posteriormente se sembraron alícuotas de la solución $(20 \mu \mathrm{L})$, dispersándola con la espátula de Drigalski sobre placas de Petri con Agar Nutritivo (Ramos et al., 2012) y se incubaron en estufa para el crecimiento de bacterias. Las colonias que crecieron en estas placas fueron aisladas por la técnica de estrías e incubadas a $30{ }^{\circ} \mathrm{C}$ por 24 a 48 horas. Este procedimiento se repitió sucesivas veces hasta obtener cepas puras de la bacteria en estudio. Bacillus subtilis fue identificada a partir de pruebas fisicoquímicas con el apoyo de claves específicas (Bergey, 1994), lo que se complementó con el análisis molecular.

\section{Aislamiento de hongos}

Las cepas de Aspergillus sección nigri y Fusarium solani fueron proporcionadas por el cepario del Laboratorio de Microbiología Agrícola y Salud Apícola y Meliponícola de la Facultad de Ciencias Agrícolas de la Universidad Nacional de Jujuy (UNJu), Argentina. Se inocularon en agar dextrosa Sabouraud y se incubaron durante 7 días a $28^{\circ} \mathrm{C}$.

\section{Actividad antifúngica}

Se enfrentaron la cepa de B. subtilis con los hongos Aspergillus sección nigri y $F$. solani. En cada una de las pruebas se colocó $1 \mathrm{~mL}$ de una suspensión con una cantidad de $10^{6}$ esporas fúngicas en $9 \mathrm{~mL}$ de agua destilada y se cultivaron sobre agar extracto de malta (MEA), enfrentándolas luego con un cultivo de B. subtilis. Los ensayos se realizaron por triplicado; como control negativo se utilizó el cultivo de las cepas fúngicas puras. Las placas se incubaron a $28^{\circ} \mathrm{C}$ por 15 días (Ruiz et al., 2019).

La actividad antagónica de las cepas bacterianas se determinó midiendo el diámetro de las colonias fúngicas en presencia de las bacterias a los 5, 10 y 15 días y el diámetro de la colonia del hongo testigo. Con las mediciones obtenidas se procedió al cálculo del porcentaje de inhibición mediante la siguiente ecuación:

$$
\mathrm{I}=[(\mathrm{C}-\mathrm{T}) / \mathrm{C}]{ }^{*} 100
$$

siendo: I la inhibición \%, C el diámetro de las colonias testigo y $\mathrm{T}$ el de las colonias tratadas (Zamora Natera et al., 2005).

A partir de los resultados obtenidos se realizó la prueba de Kruskal-Wallis para datos no paramétricos para las variables en estudio a partir del programa Infostat versión 2015 (Di Rienzo et al., 2015).

\section{RESULTADOS}

Los diámetros de las colonias de $A$. sección nigri frente a las bacterias en estudio a los 5 días de incubación fueron menores a $46 \mathrm{~mm}$ (Tabla 1, Fig. 1). Los diámetros de las colonias de F. solani a los 5 días de incubación fueron menores a $28 \mathrm{~mm}$ (Tabla 1, Fig. 2).

En la prueba de Aspergillus sección nigri frente

Tabla 1. Diámetro promedio de las colonias (mm) de Aspergillus sección nigri y Fusarium solani frente a Bacillus subtilis.

Table 1. Average collony diameters (mm) of Aspergillus section nigri and Fusarium solani against Bacillus subtilis.

\begin{tabular}{clllllll}
\hline & \multicolumn{3}{c}{ Aspergillus sección nigri } & & \multicolumn{3}{c}{ F. solani vs B. subtilis } \\
\cline { 2 - 3 } \cline { 7 - 8 } Días de incubación & $\mathbf{R} 1$ & $\mathbf{R} 2$ & $\mathbf{R} 3$ & & R1 & R2 & R3 \\
\hline $\mathrm{T}$ & 90 & 90 & 90 & & 70 & 90 & 90 \\
5 & 45,9 & 37,4 & 40,2 & & 20,2 & 23 & 20,5 \\
10 & 51,1 & 45,2 & 50,5 & & 30 & 31 & 30,2 \\
15 & 51,9 & 52,6 & 53,9 & & 34,3 & 32,9 & 31 \\
\hline
\end{tabular}

T: Testigo, R1: Repetición 1; R2: Repetición 2; R3: Repetición 3. 


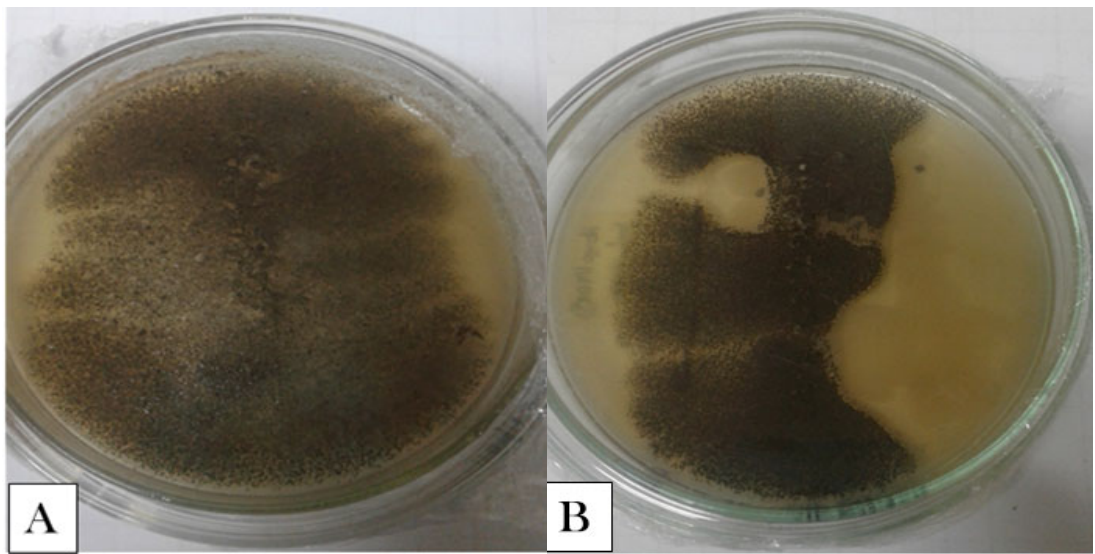

Fig. 1. (A) Testigo Aspergillus sección nigri (B) Inhibición del crecimiento ( $\mathrm{mm}$ ) de Aspergillus sección nigri frente a Bacillus subtilis a los 5 días de incubación.

Fig. 1. (A) Control Aspergillus section nigri (B) Growth inhibition (mm) of Aspergillus section nigri against Bacillus subtilis at 5 days of incubation.

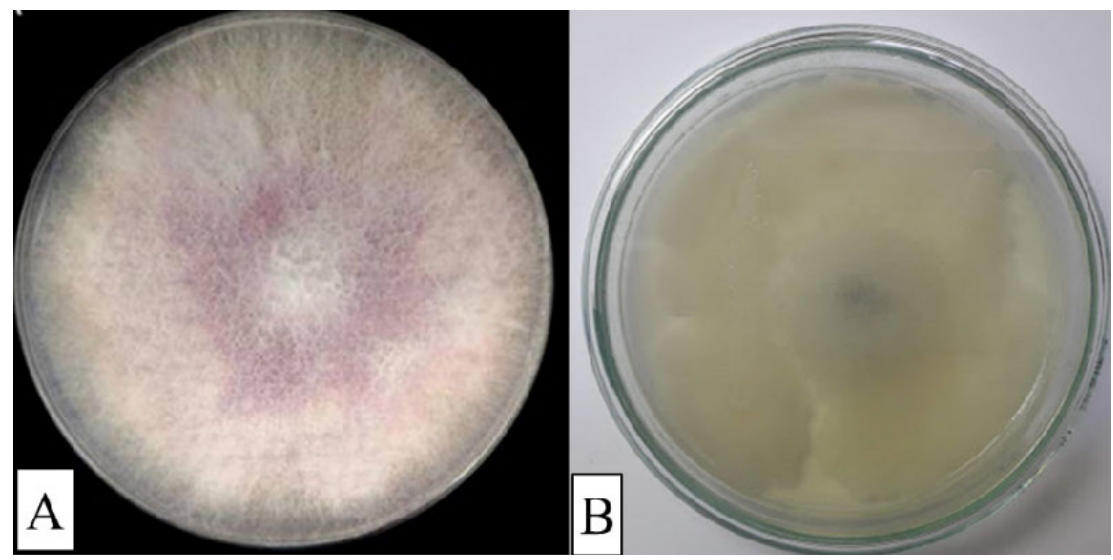

Fig. 2. (A) Testigo Fusarium solani, (B) Inhibición del crecimiento (mm) de Fusarium solani frente a Bacillus subtilis a los 10 días de incubación.

Fig. 2. (A) Control Fusarium solani, (B) Growth inhibition (mm) of Fusarium solani against Bacillus subtilis at 10 days of incubation.

a Bacillus el porcentaje de inhibición fue mayor al 50\%. El porcentaje de inhibición para Fusarium frente a Bacillus fue en promedio mayor al $67 \%$ (Tabla 2). Los porcentajes de inhibición de $A$. sección nigri y $F$. solani frente a $B$. subtilis fueron mayores a los 5 días de incubación (Tabla 2).

La prueba estadística de Kruskal Wallis realizada con el programa Infostat no arrojó diferencias significativas $(\mathrm{p} \leq 0,05 ; \mathrm{p}=0,33$; $\mathrm{gl}$ 5 ) entre los porcentajes de inhibición en los tres tiempos de incubación entre las pruebas de $F$. solani y Aspergillus sección nigri frente a B. subtilis.

\section{DISCUSIÓN}

Los resultados obtenidos mostraron que Bacillus subtilis ejerce efecto antagónico al enfrentarlos a hongos patógenos de las abejas, inhibiendo su crecimiento en diferente grado. Esta bacteria se destaca como inhibidora de crecimiento de algunos hongos y/o bacterias fitopatógenas (Pérez et al., 2015). Los mayores valores del efecto antagónico de $B$. subtilis frente a los hongos se obtuvieron en el menor tiempo probado (5 días), pero a pesar de esto no se 
Tabla 2. Inhibición del crecimiento (\%) de Aspergillus sección nigri y Fusarium solani frente a Bacillus subtilis.

Table 2. Growth inhibition (\%) of Aspergillus section nigri and Fusarium solani against Bacillus subtilis.

\begin{tabular}{|c|c|c|c|c|c|c|}
\hline \multirow[b]{2}{*}{ \% de Inhibición } & \multicolumn{3}{|c|}{$\begin{array}{c}\text { Aspergillus sección nigri vs } \\
\text { B. subtilis }\end{array}$} & \multicolumn{3}{|c|}{ F. solani vs B. subtilis } \\
\hline & R1 & $\mathbf{R} 2$ & R3 & R1 & $\mathbf{R} 2$ & R3 \\
\hline 5 días & 49 & 58 & 55 & 71 & 67 & 71 \\
\hline 10 días & 43 & 50 & 44 & 67 & 66 & 66 \\
\hline 15 días & 42 & 42 & 40 & 62 & 63 & 66 \\
\hline
\end{tabular}

R1: Repetición 1; R2: Repetición 2; R3: Repetición 3.

encontraron diferencias significativas entre los tiempos estudiados, lo que nos indica que el efecto inhibidor es persistente en el tiempo.

Los valores altos del efecto inhibidor en el menor tiempo probado podría estar relacionado con la disminución de la producción de metabolitos antifúngicos por parte de estos aislados, y/o con la producción de compuestos por parte del hongo que inhiban el crecimiento bacteriano, dado que durante los primeros días del cultivo dual, las bacterias ejercen un fuerte efecto antagónico sobre los hongos patógenos, por su alta velocidad de crecimiento, lo que les permite alcanzar rápidamente el estado estacionario y comenzar a producir metabolitos secundarios con actividad antifúngica y finalmente colonizar el medio de cultivo. Posteriormente los hongos podrían iniciar la producción de metabolitos que contrarresten el efecto producido por las bacterias antagonistas, y/o los metabolitos bacterianos pudieran disminuir su concentración en función del tiempo, lo que explicaría el resultado obtenido (Tejera et al., 2012).

A partir de las pruebas realizadas de los diferentes hongos patógenos de abejas frente a la cepa bacteriana aislada de las cutículas de hormigas de colmenas se determinó que la prueba de inhibición fue efectiva en más de un $50 \%$ para Aspergillus sección nigri como para Fusarium solani. Esto indica que en principio la cepa bacteriana puede servir de control de enfermedades micóticas de las abejas melíferas (A. mellifera) y que podría ser controladora de otros agentes etiológicos como virus, bacterias y parásitos que ocasionan daños considerables en la apicultura (Verde Jiménez et al., 2013). Principalmente la respuesta antagónica de la bacteria está asociada con la síntesis de lipopéptidos como iturina, surfactina, fengicina y bacilomina (López-Hernández, 2020). Las especies de Bacillus asociadas con abejas melíferas son una fuente interesante de compuestos bioactivos, con usos potenciales más allá del campo de la apicultura, pues la mayoría producen una amplia gama de compuestos antimicrobianos con actividad contra bacterias y hongos (Bartel et al., 2018). Por todo esto, cabe destacar la importancia de la actividad antimicrobiana de $B$. subtilis que actúa como supresor efectivo de patógenos en la sanidad de las colmenas. Dicha acción puede ser aprovechada como forma de control biológico de hongos patógenos potencialmente perjudiciales para la actividad apícola (Ruiz et al., 2019).

La presencia de enfermedades en las colmenas de abejas melíferas (A. mellifera) reduce la producción de miel, y en ciertos casos pueden ocasionar la pérdida de la colonia sino se controlan adecuadamente (Guzmán-Novoa et al., 2011). Por ello estas pruebas resultan importante para establecer un posible uso como control biológico frente a enfermedades que afectan la sanidad de las abejas.

\section{CONCLUSIÓN}

La cepa de Bacillus subtilis ejerce un efecto antagónico frente a microorganismos patógenos de abejas. Se evidencia la importancia de conocer los microrganismos presentes en hormigas que anidan las colmenas y la interacción que presentan con las abejas, los que pueden contribuir a un mejor estado de los colmenares. Los datos obtenidos aportarán a futuros estudios sobre las interacciones entre microorganismos aislados de hormigas que anidan colmenas y microorganismos patógenos de abejas, generando estrategias para un potencial control futuro de enfermedades que implique la aplicación directa de un agente de control biológico.

\section{AGRADECIMIENTOS}

A los socios de la COPAJ (Cooperativa de Productores Apícolas de Jujuy Ltda., Argentina) $\mathrm{y}$ al productor Ezequiel Verrastro. 


\section{LITERATURA CITADA}

Calle Barrientos Y. M., y J.Y. Agudelo Valencia. 2016. Identificación de patógenos causantes de enfermedades en abejas Apis mellifera en apiarios productores de miel del municipio de Marsella Departamento de Risaralda. Trabajo de Grado para el título de Zootecnista. Universidad Nacional Abierta y a distancia UNAD. Escuela de Ciencias Agrícolas, Pecuarias y del Medio Ambiente. ECAPMA. Programa Zootecnia CEAD Medellin-CCAV Dosquebradas, Risaralda. Disponible en: https://repository.unad.edu.co/jspui/ handle/10596/719/simple-search?query=\&sort_by $=$ score\&order $=$ desc $\&$ rpp $=10 \&$ filter_field_1=author\&filter_type_1=equals\&filter_value_1=Agudelo+Valencia\%2C+Jhenny + Yomara\&etal $=0$ \&filtername $=$ author\&filterquery $=$ Calle + Barrientos $\% 2 \mathrm{C}+$ Yesica + Maria\&filtertype $=$ equals

Bartel, L., E. Abrahamovich, C. Mori, A. López, and A. Alippi. 2018. Bacillus and Brevibacillus strains as potential antagonists of Paenibacillus larvae and Ascosphaera apis. Journal of Apicultural Research 58:2-16.

Bergey D.H., and J. Holt. 1994. Manual of Systematic Bacteriology. 9th. ed. Williams \& Wilkins, Baltimore, Maryland.

Castillo-Reyes, F., F. Hernández-Castillo, G. Gallegos-Morales, A. Flores-Olivas, R. Rodríguez-Herrera y C. Aguilar. 2015. Efectividad in vitro de Bacillus y polifenoles de plantas nativas de México sobre Rhizoctonia-Solani. Revista Mexicana de Ciencias Agrícolas 6(3):549-562.

Di Rienzo, J.A., F. Casanoves, M.G. Balzarini, L. Gonzalez, M. Tablada, y C.W. Robledo. 2015. Grupo InfoStat, FCA, Universidad Nacional de Córdoba, Argentina. Disponible en http:// www.infostat.com.ar (Consulta 5 de Febrero 2021).

FAO. 2018. Organización de las Naciones Unidas para la Agricultura y la alimentación. Italia. Disponible en https://www.izslt. it/apicoltura/wp-content/uploads/ sites/4/2016/03/Aspergilosis-_-TECA.pdf (Consultado el 19 de noviembre de 2021).

Guzmán-Novoa, E., A. Correa-Benítez, L.G. Espinosa-Montano y G. Guzmán-Novoa. 2011. Colonización, impacto y control de las abejas melíferas africanizadas en México. Veterinaria México (México) 42(2):149-178.

López, M.B. 2014. Bacterias y hongos en el aire interior de seis áreas de la Universidad Agraria de la Selva. Universidad Agraria de la Selva. Tingo María, Perú.
López-Hernández, M.B. 2020. Bacterias rizosféricas asociadas al cultivo de maíz (Zea mays L.) con potencial biofertilizante en el estado de Campeche. Tesis Ingeniero Agrónomo. Instituto Tecnológico de Chiná, Campeche, México. Disponible en https://rinacional.tecnm.mx/jspui/handle/ TecNM/899

Martínez-Hernández, D.G. y A. Callejas-Chavero. 2015. Efecto mutualista de Liometopum apiculatum (Hymenoptera: Formicidae) sobre la tasa de parasitoidismo en Toumeyella martinezi (Hemiptera: Coccidae) asociados a Myrtillocactus geometrizans (Cactaceae). p.109-120. En M. Vásquez-Bolaños, CastañoMeneses G., Cisneros-Caballero A., QuirozRocha G., Navarrete-Heredia, J. (eds.) FORMICIDAE DE MÉXICO. Instituto de Ecología, Cuerpo Académico de Zoología, Ciudad de México.

Pérez, Á., A. Coto, P. Echemendía, y Q.G. Ávila. 2015. Pseudomonas fluorescens Migula, ¿control biológico o patógeno? Rev. Protección Veg. 30(3):225-234.

Ramírez Del Jesús, Y. 2016. Efecto del tiempo de secado en las características fisicoquímicas, microbiológicas y sensoriales del polen de abejas (Apis mellifera), Escuela Agrícola Panamericana, Zamorano Honduras. Disponible en https://bdigital.zamorano.edu/ bitstream/11036/5780/1/AGI-2016-T037.pdf

Ramos, A.M., T. Sepúlveda, L. Prado, K. Matsumoto, F. Ramírez, y M. Salazar. 2012. Manual de prácticas de laboratorio. Microbiología General. México, Universidad Autonoma Metropolitana, Unidad Iztapalapa, México. Disponible en http:// publicacionescbs.izt.uam.mx/DOCS/ MMICROBIO_GENERAL.pdf

Ricci, M., D. Benítez, S. Padín, y A. Maceiras. 2005. Hormigas Argentinas: Comportamiento, Distribución y Control. Facultad de Ciencias Agrarias y Forestal. Universidad Nacional de la Plata, Argentina. Disponible en https://www.academia. edu/7443780/COMPORTAMIENTO_ DISTRIBUCI\%C3\%93N_Y_CONTROL

Ruiz, G.B., y M. Benítez. 2018. Registro de hormigas (Hymenoptera: Formicidae) presentes en apiarios de Apis mellifera L. de los Valles Templados de la provincia de Jujuy-Argentina. Journal of the Selva Andina Research Society 9:113-119.

Ruiz, G.B., R.M. Retamoso, y M. Benítez Ahrendts. 2019. Pruebas de inhibición de Bacillus subtilis sobre hongos transportados por hormigas invasoras de colmenas apícolas. Revista Científica Agrarias (19):87-91. 
Serrano-Coll, H.A., N. Cardona-Castro. 2015. Micotoxicosis y micotoxinas: generalidades y aspectos básicos. Rev CES Med 29(1):143-152

Tejera, B., M. Heydrich, y M. Rojas. 2012. Antagonismo de Bacillus spp. frente a hongos fitopatógenos del cultivo del arroz (Oryza sativa L.) Rev. Protección Vegetal 2:117-122.

Valega, O. 2016. Mil consejos de cómo matar una amiga. Mis amigas las hormigas, tan vapuleadas y tan útiles para el apicultor. Disponible en https://www.apiservices.biz/ es/articulos/ordenar-por-popularidad/1196mil-consejos-de-como-matar-una-amiga (Consulta 15 abril 2020).

Verde-Jiménez, M., J. Demedio-Lorenzo y T. Gómez-Bernia. 2013. Apicultura. Salud y producción. Guía técnica para el apicultor. Consejo Científico Veterinario de Cuba, Vedado, Plaza de la Revolución, La Habana, Cuba. Disponible en https://isbn. cloud/9789597190226/apicultura-salud-yproduccion-guia-tecnica-para-el-apicultor/
Villarreal-Delgado, M.F., E.D. Villa-Rodríguez, L.A. Cira-Chávez, M.I. Estrada-Alvarado, F.I. Parra-Cota y S. de los Santos-Villalobos. 2018. El género Bacillus como agente de control biológico y sus implicaciones en la bioseguridad agrícola. Mex. J. Phytopathol. 36:95-130.

Zamora Natera, J.F., A. Bernal Alcocer, M. Ruiz López, M. Soto Hernández, A. Escalante Estrada, y H. Vibrans Lindemann. 2005. Perfil de alcaloides de semillas de Lupinus exaltatus Zucc. (Fabaceae) y la evaluación antifúngica del extracto alcaloideo y lupanina contra fitopatógenos. Revista Mexicana de Fitopatología 23(2):124-129. 